\title{
A Mathematical Model for the Dynamics of Zika Virus via Homotopy Perturbation Method
}

\author{
*11ADAMU, G; ${ }^{1}$ BAWA, M; ${ }^{3}$ JIYA, M; ${ }^{4}$ CHADO, UD \\ ${ }^{1}$ Directorate of Army Training Support, Training and Doctrine Command, Minna, Nigeria \\ ${ }^{2}$ Department of Mathematics /Computer, Ibrahim Badamasi Babangida University, Lapai, \\ Nigeria \\ ${ }^{3}$ Department of Mathematics, Federal University of Technology, Minna, Nigeria \\ ${ }^{4}$ Department of Mathematics, College of Education, Minna, Nigeria \\ Correspondence Author: email; ahlul_baity@yahoo.com 07039020817
}

\begin{abstract}
Zika virus is a member of the Flavivirus genus of the Flaviviridae family, which includes other globally relevant human's pathogens such as dengue virus, yellow fever virus, West Nile virus and tick-borne encephalitis virus. In this paper, a deterministic mathematical model of Zika virus was formulated using ordinary differential equations with two control strategies: treatment for humans and insecticide spray for mosquitoes. Homotopy Perturbation Method was used to obtain the approximate solution of the model. From the result obtained, $59 \%$ effective administration of insecticide spray proved effective which showed a great reduction in the infected humans as well as infected vector population. Numerical results were offered in the form of Graphs. This research work contributes to new field of knowledge included to the dynamics of Zika virus in population's dynamics with the application of Homotopy Perturbation Method and can be further extended to study the pattern of Zika associated diseases that pose a significant public health risk. () JASEM

\section{https://dx.doi.org/10.4314/jasem.v21i4.1}

Keywords: Homotopy Perturbation method, Zika virus, Modelling, Numerical Simulations

Zika virus is a mosquito-borne flavivirus diseases that was first identified in Uganda in 1947 in monkeys through a network that monitored yellow fever. It was later identified in humans in 1952 in Uganda and Tanzania. Outbreaks of Zika virus disease have been recorded in Africa, America, Asia and the Pacific. From the 1960s to 1980 s, human infections were found across Africa and Asia, typically accompanied by mild illness. The first large outbreak of disease caused by Zika infection was reported from the Island of Yap (Federated States of Micronesia) in 2007. In July 2015 Brazil reported an association between Zika virus infection and Guillain-Barré syndrome. In October 2015 Brazil reported an association between Zika virus infection and microcephaly (WHO, 2016). Zika virus (ZIKV) is primarily transmitted to people through the bite of an infected mosquito from the Aedes genus, mainly Aedes aegypti in tropical regions. Aedes mosquitoes usually bite during the day, peaking during early morning and late afternoon/evening. This is the same mosquito that transmits dengue, chikungunya and yellow fever. Sexual transmission of Zika virus is also possible. Other modes of transmission such as blood transfusion are being investigated (Musso et al., 2015). The incubation period of Zika virus disease is not clear, but is likely to be a few days. The symptoms are similar to other infections such as dengue, fever, skin rashes, conjunctivitis, muscle and joint pain, malaise, and headache. These symptoms are usually mild and last for 2-7 days. The recent ZIKV incidence in Brazil and French Polynesia possibly compels World Health Organization (WHO) to declare a Public Health Emergency of International Concern in response to the clusters of microcephaly and other neurological disorders. Apart from the major outbreak in French Polynesia, ZIKV incident shows 42 Guillain-Barre syndrome cases between March 2014 and May 2015 in same region and severe brain lesions were reported (Ebenezer and Kazeem, 2016). Consequently, at this time there were no recognized vaccines or drugs to cure the ZIKV infection.

The Homotopy Analysis Method is one of the wellknown methods to solve the linear and non-linear equations. In the last decade, the idea of Homotopy was combined with perturbation. The fundamental work was done by $(\mathrm{He}, 2000)$. This method involves a free parameter, whose suitable choice results into fast convergence. He introduced Homotopy Perturbation Method and its application (He, 2006, 2008, 2009). These methods are independent of the assumption of small parameter as well as they cover 
all the advantages of the perturbation method. Muhammad et al. (2014) developed a model of SIR by Homotopy Perturbation Method (HPM).The models were solved by NSFD, RK4 and compared the HPM solution with NSFD and RK4, it found that the HPM solution have a good agreement with other standard method of NSFD and RK4. Abubakar et al. (2013) built a model of SIR for infectious virus dynamics, using Homotopy Perturbation Method (HPM). The analytical solution was obtained to present the model graphically, which gives the better understanding of the infectious virus dynamics. Their result shows that the reductions of disease frequency match or even supersede the infection rate.

A lot of mathematical models have been built up as a tool for investigating the dynamics transmission of infectious diseases. Ebenezer and Kazeem, (2016) formulated a mathematical model for optimal control strategies of Zika virus to examine the effect of mass treatment and insecticide, in other to minimize the number of Zika infected hosts and vectors with optimal cost of mass treatment and insecticide. Adam et al. (2014) developed a mathematical model to simulate vector-borne transmission for people and mosquitoes, the model incorporated delays as a result of the intrinsic (human) and extrinsic (vector) incubation periods. Onuorah et al. (2016) developed a deterministic model for the dynamics of Zika virus. The model incorporates vital dynamics for both the human and vector compartments. Bawa et al. (2016) developed a deterministic model of tuberculosis disease using HPM to analyze the rate of TB infections on human populations. Their result revealed that $\mathrm{TB}$ disease can be drastically reduced with $65 \%$ effectiveness in vaccine administration. The objective of this paper is to develop a mathematical model for the dynamics of zika virus using HPM with two control strategies that is, treatment for humans and insecticide spray for mosquitoes. The impact of these two strategies is to be further examined.

\section{MATERIALS AND METHODS}

The clinical symptoms of Zika virus was used to determine the model of Zika virus represented by ordinary differential equations (1) to (5). The deterministic model of Zika virus with five compartments and control strategies of both humans and vectors were formulated. The positions of ordinary differential equations of the non-linear system are as follow: The data used to test the model is on table 1 and the systems of non-linear equations are as follow:

$$
\begin{aligned}
& \frac{d S_{h}}{d t}=\wedge_{h}-\left(\mu_{h}+\beta_{h} I_{v}\right) S_{h} \\
& \frac{d I_{h}}{d t}=\beta_{h} S_{h} I_{v}-\left(\mu_{h}+\mu_{0}+\lambda+\delta_{h}\right) I_{h} \\
& \frac{d R_{h}}{d t}=\left(\lambda+\delta_{h}\right) I_{h}-\mu_{h} R_{h} \\
& \frac{d S_{v}}{d t}=\wedge_{v}-\beta_{v} S_{v} I_{h}-\left(\mu_{v}+\delta_{v}\right) S_{v} \\
& \frac{d I_{v}}{d t}=\beta_{v} S_{v} I_{h}-\left(\mu_{v}+\delta_{v}\right) I_{v}
\end{aligned}
$$

where; $S_{h}$ represents susceptible humans, $S_{v}$ is susceptible vectors, $I_{h}$ infectious humans, $I_{v}$ infectious vectors, $R_{h}$ is recovered humans, $\mu_{h}$ natural death rate of humans, $\mu_{v}$ natural death of vectors, $\delta_{v}$ represent death rate from insecticide, $\delta_{h}$ represent recovery rate from treatment, $\beta_{h}$ the spread rate from humans to mosquitoes, $\beta_{v}$ spread rate from mosquitoes to humans, $\wedge_{h}$ represents recruitment number of susceptible 
humans, $\wedge_{v}$ recruitment number of susceptible vectors, $\mu_{0}$ death due to infection, $\lambda$ probability of infectious humans being recovered

Solution of the Model Using Homotopy Perturbation Method (HPM): Homotopy Perturbation Method (HPM) was developed by $(\mathrm{He}, 2000)$. The HPM provides solution to various linear and non-linear differential equations (Jiya, 2010)

The basic ideas of the method are by considering the following non-linear differential equation:

$$
A(U)-f(r)=0, r \in \Omega
$$

with the boundary condition

$$
B\left(U, \frac{\partial U}{\partial n}\right)=0, r \in \Gamma
$$

Where A is the general differential operator, B the boundary operator, $f(r)$ is the analytical function and $\Gamma$ is the boundary of the domain $\Omega$. The A operator can be divided into two major parts $L$ and $N$ been the linear and nonlinear component respectively. Equation (6) can be written as follows:

$$
L(U)+N(U)-f(r)=0, r \in \Omega
$$

The HPM is composed as follows:

$$
H(V, h)=(1-h)\left[L(V)-L\left(U_{0}\right)\right]+h[A(V)-f(r)]=0
$$

Where $V(r, P): \Omega \in[0,1] \rightarrow R$

From equation (8), $P \in[0,1]$ is an embedded parameter and $U_{0}$ is the approximation that satisfies the boundary condition. The solution to equation (10) can be assumed as power series in $h$ as follows:

$$
V=V_{0}+h V_{1}+h^{2} V_{2}+\ldots
$$

With approximation best obtain when:

$$
\begin{gathered}
U=\lim _{h \rightarrow 1}=v_{0}+h v_{1}+h^{2} v_{2}+\ldots \\
\end{gathered}
$$

The rate of convergence majorly depends on the nonlinear operator $\mathrm{A}(\mathrm{V})$

\section{Solution of the Model Equations}

$$
\begin{aligned}
& \frac{d S_{h}}{d t}+\left(\mu_{h}+\beta_{h} I_{v}\right) S_{h}-\wedge_{h}=0 \\
& \frac{d I_{h}}{d t}+\left(\mu_{h}+\mu_{0}+\lambda+\delta_{h}\right) I_{h}-\beta_{h} S_{h} I_{v}=0 \\
& \frac{d R_{h}}{d t}+\mu_{h} R_{h}-\left(\lambda+\delta_{h}\right) I_{h}=0 \\
& \frac{d S_{v}}{d t}+\beta_{v} S_{v} I_{h}+\left(\mu_{v}+\delta_{v}\right) S_{v}-\wedge_{v}=0 \\
& \frac{d I_{v}}{d t}+\left(\mu_{v}+\delta_{v}\right) I_{v}-\beta_{v} S_{v} I_{h}=0
\end{aligned}
$$


With the following initial conditions $S_{h}(0)=S_{0}, I_{h}(0)=I_{0}, R_{h}(0)=R_{0}, S_{v}(0)=\phi_{0}$ and $I_{v}(0)=\tau_{0}$

Let

$$
\begin{aligned}
& S_{h}=a_{0}+h a_{1}+h^{2} a_{2}+\ldots \\
& I_{h}=b_{0}+h b_{1}+h^{2} b_{2}+\ldots \\
& R_{h}=c_{0}+h c_{1}+h^{2} c_{2}+\ldots \\
& S_{v}=d_{0}+h d_{1}+h^{2} d_{2}+\ldots \\
& I_{v}=e_{0}+h e_{1}+h^{2} e_{2}+\ldots
\end{aligned}
$$

Applying Homotopy Perturbation Method (HPM) to equations (12) - (16) using equations (17) - (21), we obtained the orders of $\mathrm{h}$ as follows:

$$
\begin{aligned}
h^{0}: & a_{0}^{\prime}=0, b_{0}^{\prime}=0, c_{0}^{\prime}=0, d_{0}^{\prime}=0 \text { and } e_{0}^{\prime}=0 \\
h^{1}: & a_{1}^{\prime}+\mu_{h} a_{0}+\beta_{h} a_{0} e_{0}-\wedge_{h}=0 \\
& b_{1}^{\prime}+\alpha b_{0}+\beta_{h} a_{0} e_{0}=0 \\
& c_{1}^{\prime}+\mu_{h} c_{0}-\left(\lambda+\delta_{h}\right) b_{0}=0 \\
& d_{1}^{\prime}+\beta_{v} b_{0} d_{0}+\left(\lambda+\delta_{v}\right) d_{0}-\wedge_{v}=0 \\
& e_{1}^{\prime}+\left(\mu_{v}+\delta_{v}\right) e_{0}-\beta_{v}\left(b_{0} d_{0}\right)=0 \\
h^{2}: & a_{2}^{\prime}+\mu_{h} a_{1}+\beta_{h}\left(a_{0} e_{1}+a_{1} e_{0}\right)=0 \\
& b_{2}^{\prime}+\alpha b_{1}+\beta_{h}\left(a_{0} e_{1}+a_{1} e_{0}\right)=0 \\
& c_{2}^{\prime}+\mu_{h} c_{1}-\left(\lambda+\delta_{h}\right) b_{1}=0 \\
& d_{2}^{\prime}+\beta_{v}\left(b_{0} d_{1}+b_{1} d_{0}\right)+\left(\mu_{v}+\delta_{v}\right) d_{1}=0 \\
& e_{2}^{\prime}+\left(\mu_{v}+\delta_{v}\right) e_{1}-\beta_{v}\left(b_{0} d_{1}+b_{1} d_{0}\right)=0
\end{aligned}
$$

where $\alpha$ is denoted as $\mu_{h}+\mu_{0}+\lambda+\delta_{h}$

Solving equations (22) - (24) using the initial condition and collecting the coefficients of power of ' $h$ ' by expansion, we obtained:

$$
\begin{aligned}
& h^{0}: a_{0}=S_{0}, b_{0}=I_{0}, c_{0}=R_{0}, d_{0}=\phi_{0} \text { and } e_{0}=\tau_{0} \\
& h^{1}: a_{1}(t)=\left(-S_{0} \tau_{0} \beta_{h}-S_{0} \mu_{h}+\wedge_{h}\right) t \\
& b_{1}(t)=\left(-S_{0} \tau_{0} \beta_{h}-\alpha I_{0}\right) t \\
& c_{1}(t)=\left(\lambda I_{0}+I_{0} \delta_{h}-R_{0} \mu_{0}\right) t \\
& d_{1}(t)=\left(I_{0} \phi_{0} \beta_{v}-\mu_{v} \phi_{0}-\phi_{0} \delta_{v}+\wedge_{v}\right) t \\
& e_{1}(t)=\left(I_{0} \phi_{0} \beta_{v}-\mu_{v} \tau_{0}-\tau_{0} \delta_{v}\right) t
\end{aligned}
$$




$$
\begin{gathered}
h^{2}: a_{2}=-\frac{1}{2} t^{2}\left\{\begin{array}{l}
S_{0} \mu_{h}^{2}+\wedge_{h} \mu_{h}+\beta_{h} I_{0} S_{0} \phi_{0} \beta_{v}-\beta_{h} S_{0} \mu_{0} \tau_{v} \\
-\beta_{h} S_{0} \tau_{v} \delta_{v}-S_{0} \tau_{0}^{2} \beta_{h}^{2}+\beta_{h} \tau_{0} \wedge_{h}
\end{array}\right\} \\
b_{2}=-\frac{1}{2} t^{2}\left\{\begin{array}{l}
-\alpha S_{0} \tau_{0} \beta_{h}+\alpha S_{0} \mu_{h}+\wedge_{h} \alpha+\beta_{h} I_{0} S_{0} \phi_{0} \beta_{v} \\
-\beta_{h} S_{0} \mu_{v} \tau_{0}-\beta_{h} S_{0} \tau_{0} \delta_{v}-S_{0} \tau_{0}^{2} \beta_{h}^{2}+\beta_{h} S_{0} \mu_{h} \tau_{0} \\
+\beta_{h} \tau_{0} \wedge_{h}
\end{array}\right\} \\
c_{2}=-\frac{1}{2} t^{2}\left\{\begin{array}{l}
\lambda S_{0} \tau_{0} \beta_{h}+\beta_{h} S_{0} \tau_{0} \delta_{h}+\lambda I_{0} \mu_{h}-\lambda S_{0} \mu_{h}+I_{0} \mu_{h} \delta_{h} \\
+R_{0} \mu_{h}^{2}-S_{0} \mu_{h} \delta_{h}-\lambda \wedge_{h}-\wedge_{h} \delta_{h}
\end{array}\right\} \\
d_{2}=\frac{1}{2} t^{2}\left\{\begin{array}{l}
I_{0}^{2} \phi_{0} \beta_{v}^{2}+2 \beta_{v} I_{0} \mu_{v} \phi_{0}+2 \beta_{v} I_{0} \phi_{0} \delta_{v}-\beta_{v} I_{0} \wedge_{v} \\
+\beta_{v} S_{0} \phi_{0} \tau_{0} \beta_{h}-\beta_{h} S_{0} \mu_{h} \phi_{0}-\beta_{v} \phi_{0} \wedge_{h}+\mu_{v}^{2} \phi_{0} \\
+2 \mu_{v} \phi_{0} \delta_{v}+\phi_{0} \delta_{v}^{2}-\mu_{v} \wedge_{v}-\wedge_{v} \delta_{v}
\end{array}\right\} \\
e_{2}=-\frac{1}{2} t^{2}\left\{\begin{array}{l}
I_{0}^{2} \phi_{0} \beta_{v}^{2}+2 \beta_{v} I_{0} \mu_{v} \phi_{0}+2 \beta_{v} I_{0} \phi_{0} \delta_{v}-\beta_{v} I_{0} \wedge_{v} \\
+\beta_{v} S_{0} \phi_{0} \tau_{0} \beta_{h}-\beta_{v} S_{0} \mu_{h} \tau_{0} \phi_{0}-\beta_{v} \phi_{0} \wedge_{h}-\mu_{v}^{2} \tau_{0} \\
-2 \mu_{v} \tau_{0} \delta_{v}-\tau_{0} \delta_{v}^{2}
\end{array}\right\}
\end{gathered}
$$

Substituting the values into the series in equations (17) - (21) taking the $\lim$ as $h \rightarrow 1$, we obtained the result as follows:

$$
\begin{aligned}
& S_{h}(t)=S_{0}+\left(-S_{0} \tau_{0} \beta_{h}+S_{0} \mu_{h}+\wedge_{h}\right) t \\
& -\frac{1}{2} t^{2}\left\{\begin{array}{l}
S_{0} \mu_{h}^{2}+\wedge_{h} \mu_{h}+\beta_{h} I_{0} S_{0} \phi_{0} \beta_{v}-\beta_{v} S_{0} \mu_{v} \tau_{0} \\
-\beta_{h} S_{0} \tau_{0} \delta_{v}-S_{0} \tau_{0}^{2} \beta_{h}^{2}+\beta_{h} \tau_{0} \wedge_{h}
\end{array}\right\} \\
& \begin{aligned}
I_{h}(t) & =I_{0}+\left(-S_{0} \tau_{0} \beta_{h}+S_{0} \mu_{h}+\wedge_{h}\right) t
\end{aligned} \\
& -\frac{1}{2} t^{2}\left\{\begin{array}{l}
-\alpha S_{0} \tau_{0} \beta_{h}+\alpha S_{0} \mu_{h}+\wedge_{h} \alpha+\beta_{h} I_{0} S_{0} \phi_{0} \beta_{v} S_{0} \mu_{v} \tau_{0}-\beta_{h} S_{0} \tau_{0} \delta_{v}-S_{0} \tau_{0}^{2} \beta_{h}^{2}+\beta_{h} S_{0} \mu_{h} \tau_{0} \\
+\beta_{h} \tau_{0} \wedge_{h}
\end{array}\right\} \\
& \left.\begin{array}{rl}
R_{0}(t) & =R_{0}+\left(\lambda I_{0}+I_{0} \delta_{h}+R_{0} \mu_{h}\right) t \\
& \frac{1}{2} t^{2}\left\{\begin{array}{l}
\lambda S_{0} \tau_{0} \beta_{h}+S_{0} \tau_{0} \beta_{h} \delta_{h}+\lambda I_{0} \mu_{h}-\lambda S_{0} \mu_{h} \\
+I_{0} \mu_{h} \delta_{h}+R_{0} \mu_{h}^{2}-S_{0} \mu_{h} \delta_{h}-\lambda \wedge_{h} \wedge_{h} \delta_{h}
\end{array}\right\} \\
S_{v}(t)= & \phi_{0}+\left(-I_{0} \phi_{0} \beta_{v}-\mu_{v} \phi_{0}-\phi_{0} \delta_{v}+\wedge_{v}\right) t
\end{array}\right\} \\
& +\frac{1}{2} t^{2}\left\{\begin{array}{l}
I_{0}^{2} \phi_{0} \beta_{v}^{2}+2 \beta_{v} I_{0} \mu_{v} \phi_{0}+2 \beta_{v} I_{0} \phi_{0} \delta_{v}-\beta_{v} I_{0} \wedge_{v} \\
+\beta_{v} S_{0} \phi_{0} \tau_{0} \beta_{h}-\beta_{v} S_{0} \mu_{h} \phi_{0}-\beta_{v} \phi_{0} \wedge_{h}+\mu_{v}^{2} \phi_{0} \\
+2 \mu_{v} \phi_{0} \delta_{v}+\phi_{0} \delta_{v}^{2}-\mu_{v} \wedge_{v}-\wedge_{v} \delta_{v}
\end{array}\right\}
\end{aligned}
$$




$$
\begin{aligned}
I_{v}(t)=\tau_{0}+\left(I_{0} \phi_{0} \beta_{v}-\mu_{v} \tau_{0}-\tau_{0} \delta_{v}\right) t \\
-\frac{1}{2} t^{2}\left\{\begin{array}{l}
I_{0}^{2} \phi_{0} \beta_{v}^{2}+2 \beta_{v} I_{0} \mu_{v} \phi_{0}+2 \beta_{v} I_{0} \phi_{0} \delta_{v} \\
-\beta_{v} I_{0} \wedge_{v}+\beta_{v} S_{0} \phi_{0} \tau_{0} \beta_{h}-\beta_{v} S_{0} \mu_{h} \phi_{h} \\
-\beta_{v} \phi_{0} \wedge_{h}-\mu_{h}^{2} \tau_{0}-2 \mu_{v} \tau_{0} \delta_{v}-\tau_{0} \delta_{v}^{2}
\end{array}\right\}
\end{aligned}
$$

Numerical Simulations: The simulations were carried out using the following variables and parameters for initial conditions at time (t). Computations were run in maple17 software for investigation.

Table1. Parameter Descriptions, Values and Source used in simulations

\begin{tabular}{llll}
\hline Parameter $\quad$ Description & Value & Source \\
\hline$\mu_{h}$ & Natural death rate of human & 0.0008 & Assumed \\
$\mu_{v}$ & Natural death rate of vector & 0.0714 & Mojumder, et al.(2016) \\
$\delta_{v}$ & Death rate from insecticide & 0.59 & Assumed \\
$\delta_{h}$ & Recovery rate from treatment & 0.05 & Assumed \\
$\beta_{h}$ & Probability of human to mosquitoes getting infected & 0.5 & Ebenezer \& Kazeem (2016) \\
$\beta_{v}$ & Probability of mosquitoes to human getting infected & 0.005 & Assumed \\
$\wedge_{h}$ & Recruitment number of susceptible human & 100 & Ebenezer \& Kazeem (2016) \\
$\wedge_{v}$ & Recruitment number of susceptible vector & 1000 & Ebenezer \& Kazeem (2016) \\
$\mu_{0}$ & Death rate due to infectious & 0.5 & Assumed \\
$\lambda$ & Probability of human recovered from infection & 0.03 & Assumed \\
$S_{h}$ & Susceptible human & 11000 &,$"$ \\
$I_{h}$ & Infectious human & 500 &, \\
$R_{h}$ & Recovered human & 200 &, \\
$S_{v}$ & Susceptible vector & 3000 &, \\
$I_{v}$ & Infectious vector & 300 & Assumed \\
\hline
\end{tabular}

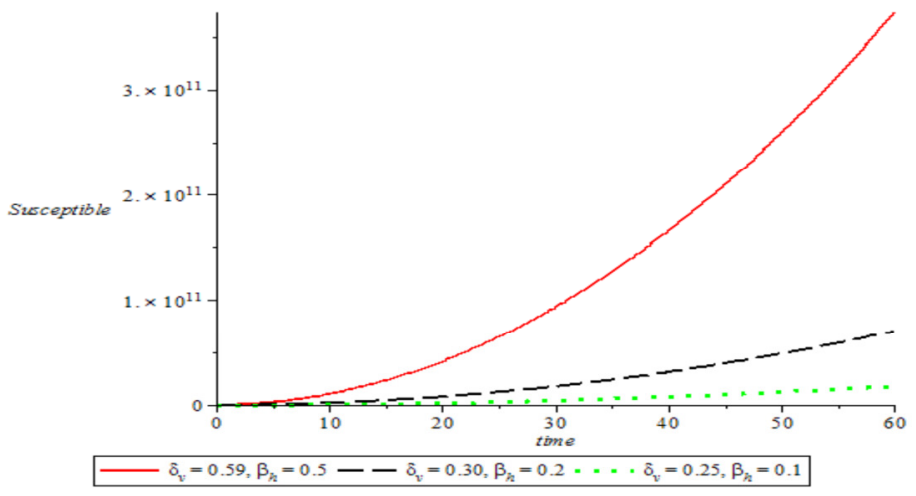

Fig 1: Graph of Susceptible humans against time with different rate of death from insecticide $\delta_{v}$ and humans to mosquitoes getting infected $\beta_{h}$. 


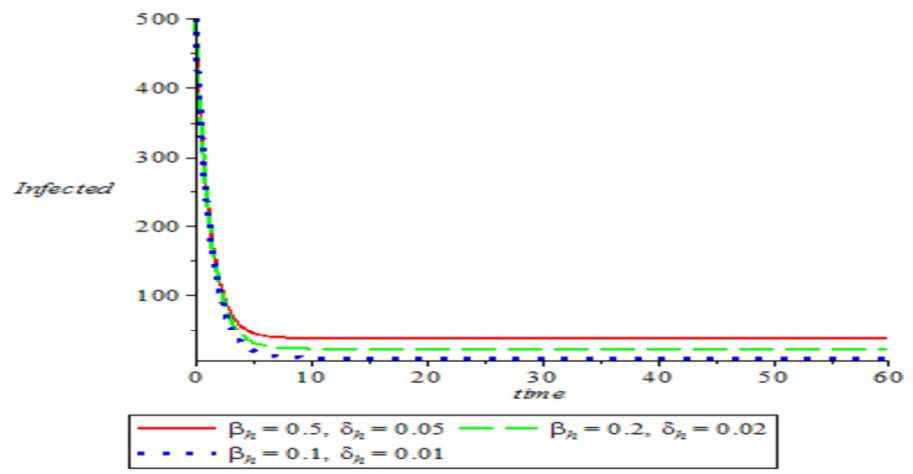

Fig 2: Graph of Infected humans against time with different rate of humans to mosquitoes getting infected $\beta_{h}$.

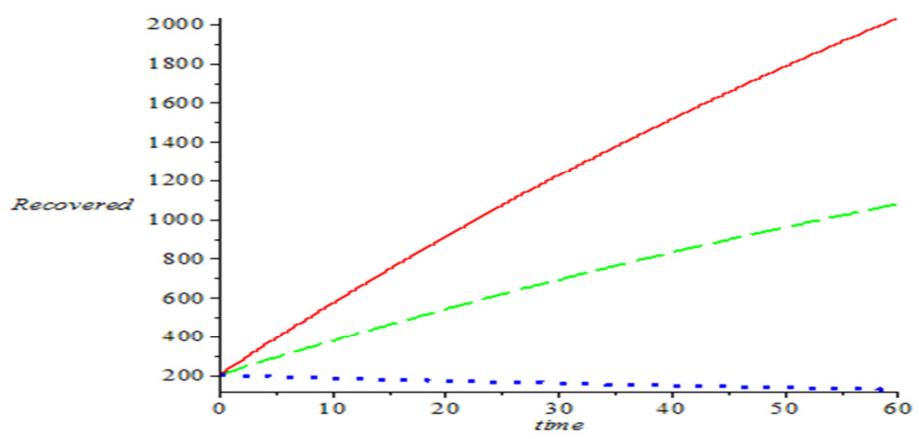

$\longrightarrow \lambda=0.03, \delta_{h}=0.05--\lambda=0.02, \delta_{h}=0.02 \cdots \lambda=0.01, \delta_{h}=0.01$

Fig 3: Graph of Recovered humans from infection against time with different rate.

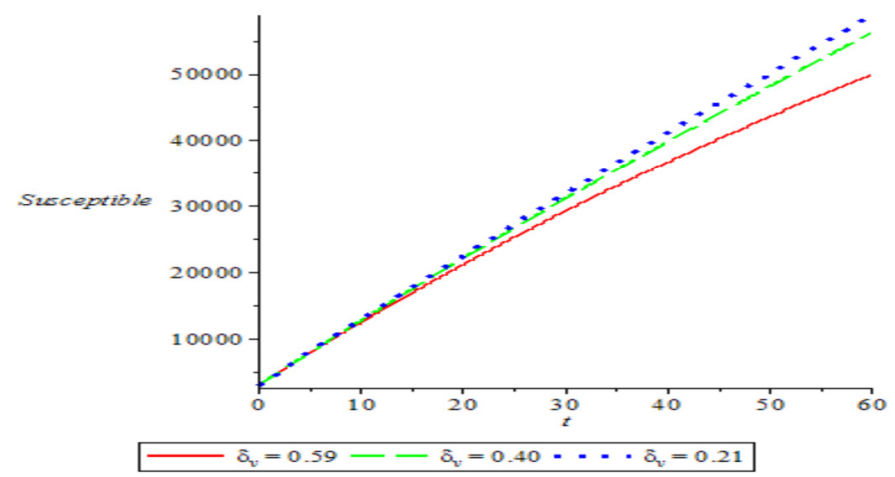

Fig 4: Graph of Susceptible vectors against time with different rate of death from insecticide $\delta_{v}$

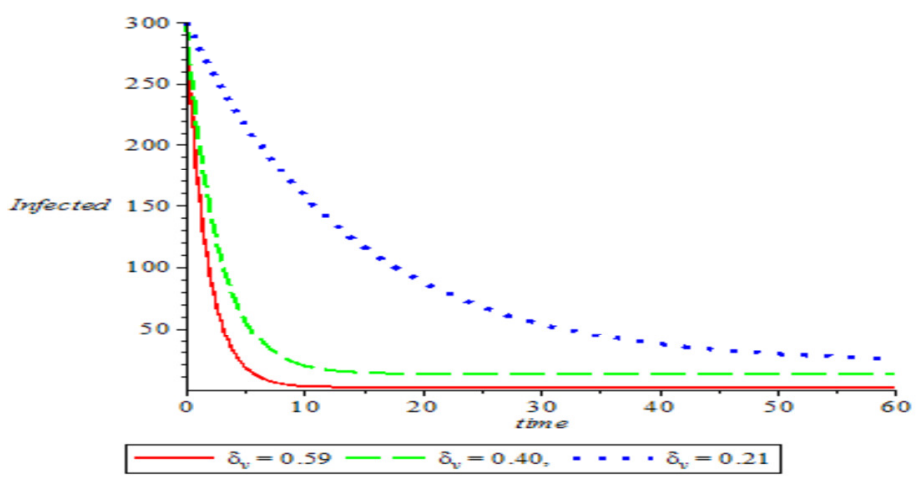

Fig 5: Graph of Infected vectors against time with different rate. 


\section{RESULTS AND DISCUSSION}

We used Homotopy Perturbation Method to determine the approximate solution of our model formulated. Mathematical software Maple17 specially designed for this purpose was used for the simulations which always give the best output. The graph in figure 1 shows the various trends for the susceptible population as the parameter values were varied. For $\delta_{v}=0.59, \beta_{h}=0.5$, the susceptible population experienced significant increase over time, this could be attributed to $59 \%$ effective use of insecticide. This can be observed in figure 5, where the infected vector population diminishes very fast particularly for $\delta_{v}=0.59$. For $\delta_{v}=0.30, \beta_{h}=0.2$ gave a fall on the susceptible population signalling eminent danger. The graph for, $\delta_{v}=0.25, \beta_{h}=0.1$, signifies that the susceptible population is most likely to go extinct if no measure is taken.

The corresponding infected population is in figure 2.The three profiles show significant decline in the infected class with the graph for $\beta_{h}=0.1$ and $\delta_{h}=$ 0.01 giving the best fit. This could be further explained from figure 4 , the graph of susceptible vector population which leads to decline in the infected human compartment. Consequently, the recovered rate increased as depicted in figure 3 . The graph for $\delta_{h}=0.05$ gives the best fit of this case.

Conclusion: In this paper, the research work contributes to new field of knowledge inclined to the dynamics of Zika virus in population dynamics with the applications of Homotopy Perturbation Method. The result of this work can further be extended to other field of knowledge to study the pattern of Zika associated diseases in many part of the world which pose a significant public health risk. It can stimulate expedited research required to address open questions and to better inform countermeasure development and clinical management.

\section{REFERENCES}

Abubakar, S;Akinwande, NI; Jimoh, OR; Oguntolu, FA; Ogwumu, OD (2013). Approximate Solution of SIR Infectious Disease Model Using Homotopy Perturbation Method (HPM).The Pacific Journal of Science and Technology, 14.(2):163-169.

Adam, JK; Sebastian, F; Rosalind, ME; Henri-Pierre, MW; John, E; Eric, JN (2014).
Transmission dynamics of Zika virus in island populations: A Modelling Analysis of the French Polynesia outbreak. doi: Org/10.1101/038588: 115.

Bawa, M; Adamu, G; Omatola, IU; Jibril, U;Yusuf, I (2016).A Mathematical Analysis of Tuberculosis Disease Population Dynamics Using Homotopy Perturbation Method, Journal of the Nigerian Association of Mathematical Physics (NAMP), ISSN:1116-4336. (37): 481-488.

Ebenezer, O; Kazeem, OO ( 2016). Mathematical Modeling of Zika virus. Asian Pacific Journal of Tropical Disease, doi: 10.1016/S22221808(16)61108-8, 6(9): 673-679.

He, JH (2008). Recent development of the homotopy perturbation method, Topological methods in nonlinear Analysis. 31(2): 205-209.

He, JH (2009). An elementary introduction to the homotopy perturbation method. Computation Maths, App, 57 (3): 410-412.

He, JH (2006). An elementary introduction to recently developed asymptotic methods and nano-mechanics in textile engineering, Int. J. Mod. Phys. B. 22(21): 3487-3578.

He, JH (2000). A coupling method of Homotopy technique and perturbation technique for nonlinear problems, International Journal of Non-Linear Mechanic, 35(1): 37-43.

Jiya, M (2010). Application of Homotopy Perturbation Method for the Solution of some Non Linear Differential Equations: Pacific Journal of Science and Technology, 11(2): 268272.

Mojumder, MS; Cohn, E; Fish, D; Brownstein, JS (2016). Estimating a feasible serial interval range for Zika fever: Bulletin of the World Health Organization, Geneva. Retrieved from,

http://www.who:16-171009.

Muhammad, AK; Syed, FS; Sher, AK; Saeed, I; Farooq, A (2014). Application of Homotopy Perturbation Method to an SIR Epidemic Model. Journal of Applied Environmental and Biological Sciences, ISSN: 2090-4274 4(3): 4954. 
Musso, D; Roche, C; Robin, E; Nhan, T; Teissier, A; Cao-Lormeau,VM (2015). Potential Sexual Transmission of Zika Virus. Emerging infectious diseases, 21(2): 359, doi: 10.3201/eid 2102.141363 PMID 2562587.

Onuorah, MO; Ademu, A; Obi, EI; Hasheem, AM (2016). Deterministic Model of Zika
Virus. Research Journali's Journal of Mathematics, ISSN: 2359-5375, 3(3): 1-15.

WHO, (2016). Retrieved from virushhttp://www.who.int/mediacentre/factsheets /zika/en/. 